\title{
106. On the Effect of Parabiosis upon the Scale Content of Wings of Each Sex in Pieris rapae crucivola Boisd
}

\author{
By Nobumasa Y AGI \\ Biology and Entomology Laboratory, Faculty of Sericulture and \\ Textile, Shinshu University, Ueda
}

(Comm. by Yô K. OKADA, M.J.A., June 12, 1954)

Several parabiotic experiments ${ }^{122334)}$ have been reported concerning the hormonal effect on the development or metamorphosis of insect.

However, it is hard to refer a paper which dealt with the effect of parabiosis on the substantial change in the content of scale of the wing to either side of sex considering the sex hormone or the artificial gynandromorphic condition. The author fortunately happened to know in the research*' on the sexual difference of scale content of

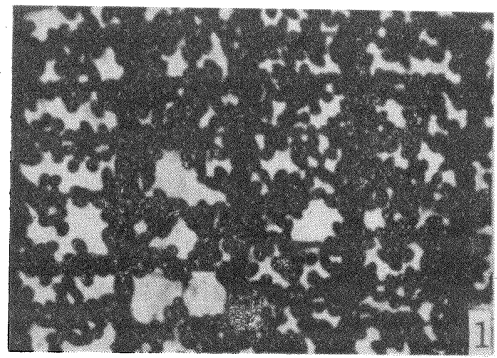

Fig. 1

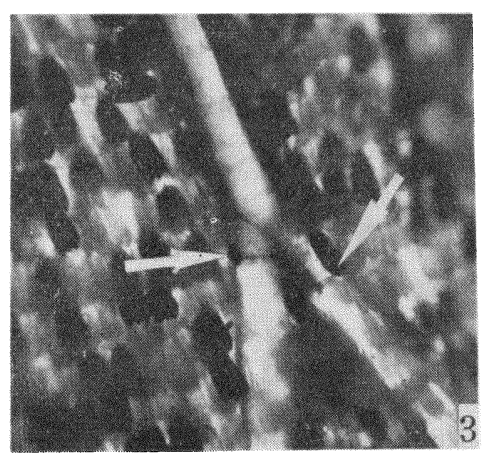

Fig. 3

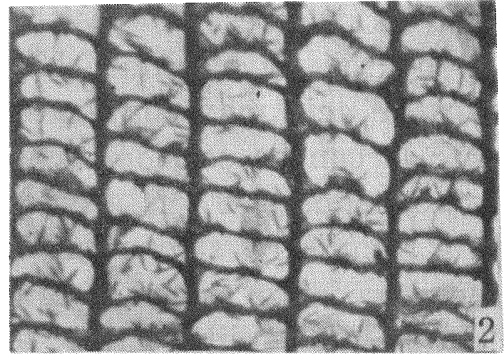

Fig. 2

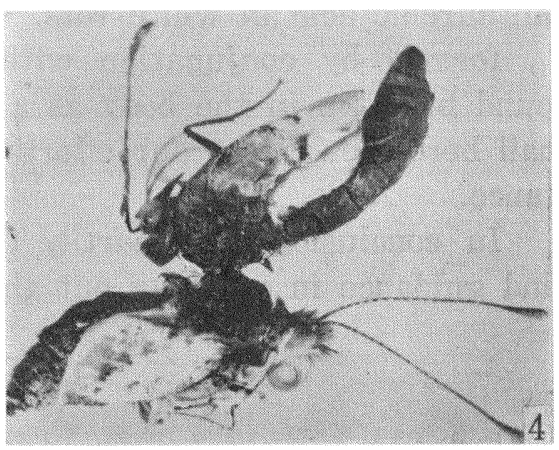

Fig. 4

Fig. 1. A part of scale in normal of wing. $\times 12000$

Fig. 2. A part of scale in normal $f$ wing. $\times 12000$

Fig. 3. A part of cubital vein in hind-wing, white arrows indicate the boundary between upper fulral reaction and under no reaction. $\times 600$

Fig. 4. Parabiotic adult, the under $f$ and the upper $\hat{o}$

*) The other paper in preparation. 
pterin derivatives in wings of Family Pieridae, that the scale of Pieris rapae crucivola has a distinct sexual difference in the formation of macromolecule of leucopterin and crystal of urea respectively in male and in female wings. These special features of sexual differences lead him to study the effect of parabiosis on the content of scale in either side of sex through the development in a union which is supplied by common blood in the pupal stage.

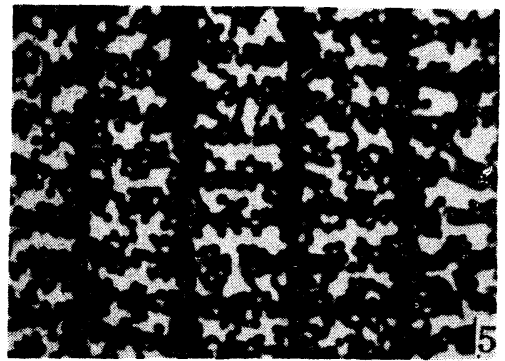

Fig. 5

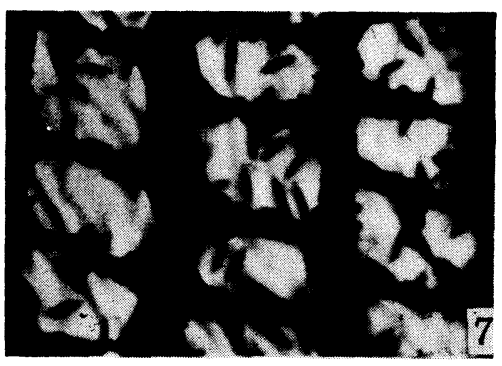

Fig. 7

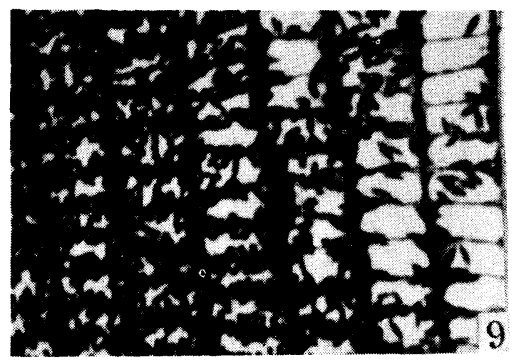

Fig. 9

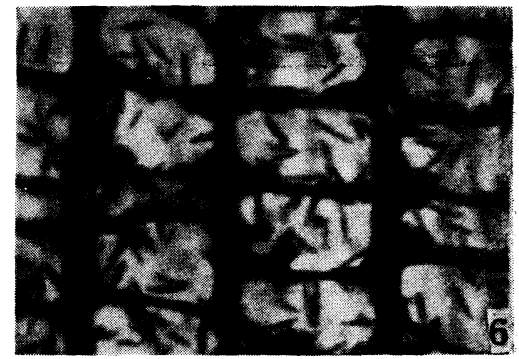

Fig. 6

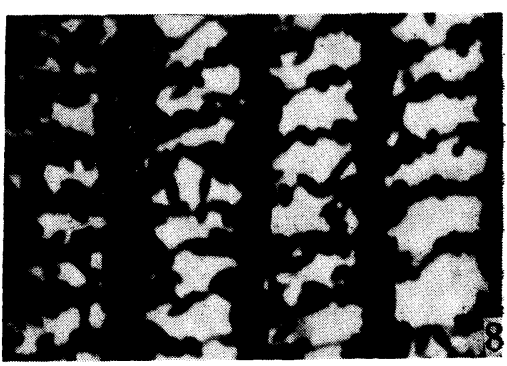

Fig. 8

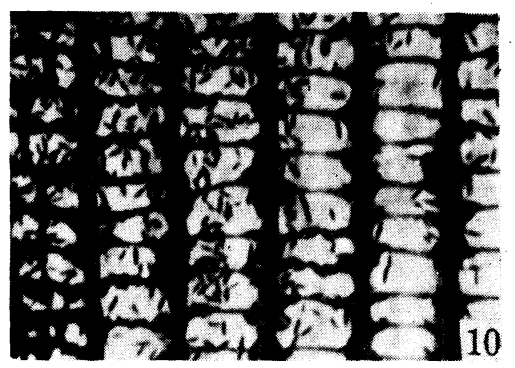

Fig. 10

Fig. 5. A central part of a scale of parabiotic of fore-wing. $\times 12000$

Fig. 6. Ditto of $q$ fore-wing. $\times 15000$

Fig. 7. A proximal part of a scale of $\hat{\delta}$ fore-wing showing extreme case (new type). $\times 14000$

Fig. 8. Ditto of $q$ fore-wing showing a new type. $\times 14000$

Fig. 9. A proximal part of a scale of $\delta$ hind-wing showing intermixing of two characters. $\times 12000$

Fig. 10. Ditto of + hind-wing showing a modification of urea crystals. $\times 12000$ 


\section{Differences in the Scale of Normal Sexes}

As it has already been reported ${ }^{5)}$ that the wing of Pieris rapae contains leucopterin and xanthopterin, the author's test by paperchromatography also proved the existence of the same substances besides leucopterin $\mathrm{B}^{6}$ in both sexes. In the scale of male wing there are globular granules which are connected with each other in a zigzag fashion from the chitinous septum of the scale (Fig. 1). Granules are quite homogeneous measuring about $1000 \AA$ in diameter and according to their molecular composition they are assumed to be an aggregated macromolecule of leucopterin which may have four junctions, correspondingly the accumulation of the same leucopterin molecule produces a macromolecule which also exhibits the tetrahedral connection as FREY-WYSSLING ${ }^{7 /}$ has suggested such kind in the coherence of the colloid particles. In the scale of female no such connected macromolecules are found except a few macromolecules on the septum, but numerous fine needle shaped crystals are distributed in the scale freely or attaching one end to the septum of the scale as if they were replacing the leucopterin macromolecules in the scale of male wing (Fig. 2). These crystals are urea derived from the blood which had been circulated in the wing vein from the pupal stage till the emergence of the adult. The residual trace of urea which had been left at the wall of the wing vein is indicated by the colour reaction of fulfral solution ${ }^{8)}$ introduced into the cubital-vein from the basal part of the hindwing (Fig. 3).

As HopkIN's assumption ${ }^{9)}$ was correct in the case of pigmentation on the colour of wings of Pieridae, the female of Pieris rapae is more active for protein metabolism in the late pupal stage to produce abundant ovum than the male in the stage of sperm formation and consequently the former produces urea as an atmost end product of protein metabolism, the latter ends in the production of leucopterin and its derivatives.

\section{Experiment of Parabiosis}

Two parabiotic adults were grown healthfully from the chrysalis with the same developmental velocity and emerged by getting help to peel off the skin at the dorsal side (Fig. 4). It is certain that the growth and metamorphic hormones were circulated homogeneously into both sexes since the union of two bodies had been performed healthfully, because both sexes developed synchronously. 
In nature, the appearance of the male is about one week earlier than that of the female. Soon after they have emerged, the male excreted a drop of brownish fluid from the terminal part, but the female did not show any excreting substances from the anus.

The external genitalia of both sexes were quite normal. At a glance of both, we could identify either sex by the characteristic colour tone and dark patterns of the wings, because the wing of the female was tinted with a little rusty white and showed two black patterns. These sexual differences in appearances tell us that the external chitinous development had been controlled in the earlier stage in the embryonic development, so the surface colour of the scale and pattern showed the ordinary sex differences as described above. But the plasmatic contents were influenced by the circulation of blood from the opposite sex as described in the following.

\section{Change of Scale Content by Parabiosis}

The scale contents from the wings of each sex were studied by electron microscope. It showed astonishingly clear pictures of many phases of intermixing of characteristic macromolecule or crystalline formation in relation to sex on either side.

On the Fore-Wing: The majority of the scale from the central to the distal part of the wing retained principally a type of male and female contents (Figs. 5, 6), but some scales from the proximal part of the wing showed a different feature as if the contents of opposite sex were introduced in the basal part of the scale as shown in Figs. 7 and 8. In these examples the scale of male site contained staut spindle shaped crystals which might have been introduced from the opposite female blood and its own leucopterin macromolecules were attached on the septum of the scale. On the contrary to the male site, the scale of female contained leucopterin macromolecules distributed over the chitinous septum as in the case of the male wing adding spindle shaped crystals somewhere at the septum. In the latter case there is the gradation on the formation of leucopterin macromolecules on the septum from single linear or zigzag attachment to manifold complex connection as seen in the normal male wing (Fig. 8, from right side to left).

On the Hind-Wing: The influence of parabiotic circulation of the blood was lesser in the hind-wing than in the fore-wing. It was indicated in the scale of the male wing in which the content of leucopterin macromolecules was partially replaced by the spindle shaped crystals gradually from basal or peripheral part towards the distal part of the scale (Fig. 9). 
The scale of female contained the spindle shaped crystals in the hind-wing without mixing of both sex characters and held nearly the female characteristics, but the crystal, which takes the stauter shape than that of scale of non-parabiotic bodies described above is caused by reception of substrates (leucopterin) produced in the body of opposite sex that must have its own metabolism depending upon the sexual differentiation especially in formation of spermatozoa in the male (Fig. 10). Certainly the blood of female contains much more urea than that of male as previously noticed. The urea is believed to be very liable to penetrate the cell membrane, according to the environmental $\mathrm{pH}$ value which has been estimated by DRAWERT ${ }^{10)}$ in the plant cells. At any rate it is stated that the urea is found in the scale of normal female of Pieris rapae and in the scale of parabiotic bodies, and in the latter the shape is modified by attachment of leucopterin on its surface resulting a stauter spindle shape. The proper type of aggregation of leucopterin molecules is a conical shape by the role of molecular composition and electric polarity, then it coacervates into gigantic macromolecules taking a zigzag connection when they grow freely, but if there is a certain foundation like a urea crystal or a fibrous substance, they grow along the line of these basal substances showing a beads-like fashion.

\section{Discussion}

Pieris rapae crucivola is found to be one of the most convenient species for parabiotic experiment to study the effect of blood from opposite sex upon the scale content of the wings of contraposite sex according to the distinct difference of plasmatic content in the scale of both sexes. The white scale from the wing of male contains leucopterin macromolecules while the scale of female contains crystals of urea being clearly photographed by an electronmicroscope. In this account it is ascertained that the purine derivatives contained in the blood of male site are capable of diffusing into the trichogenous cell of scale of female, on the contrary, urea in the blood of female enters into the scale of male site, accordingly there happens a modification of the proper feature of macromolecule of leucopterin in male and crystal of urea in female respectively. These changes caused by intermixing of blood from both sexes are not merely a mixture of substrates from both sexes. The crystal of urea in female site becomes stauter than that in the usual female scale by adhering leucopterin molecule on its surface and majority of them take a shape of slightly notching. 
In the extreme case in which the contents of both sites look like exchanged, it showed rather a new type by being composed of two elements from both sexes as was found in the gynandromorphs in Habrobracon ${ }^{11)}$ and in Drosophila. ${ }^{12)}$ In the parabiotic experiments the external sexual character of scale could not be changed as in the case of sexual cuticular character (setae) in gynandromorphs. ${ }^{22}$ In taking account of WHITING and HANNAH's observations on gynandromorphs into the author's parabiotic experiment, there is no doubt that the sexuality of external chitinous construction of insect is determined primarily in the stage of embryonic development as GoLDSCHMIDT ${ }^{13)}$ and others ${ }^{14)}$ have already supposed. In the post embryonic development it is hard to change the external sexual character by substantial exchange of plasmatic content corresponding to the hypodermal layer, though the diffusion of direct contact of cells or common circulation of blood acted. It is plausible that the biochemical or enzymatic functions of sex-chromosome are limited within each unit cell, therefore the characteristic feature of macromolecular or crystal formation in cytoplasm is differentially exhibited according to the sex, and no sex-hormonal effect through blood is observed.

The author expresses his hearty thanks to Dr. Yô K. OKADA and Prof. K. TAKEWAKI for their kind advices and to Mr. M. SAWAZI for his assistance in this research of electronmicroscope.

\section{References}

1) Bodenstein, D.: J. Exp. Zool., 123, No. 2, 189-232 (1953).

2) Fukuda, S.: Lecture "On the Parabiosis of Silk Worm", at the Ann. Meeting of Sericulture (1954).

3) Wigglesworth, V. B.: J. Exp. Biol., 17, 201-222 (1940).

4) Williams, C. W.: Biol. Bull., 103, No. 1, 120-138 (1952).

5) Wieland, H. and Purrmann, R.: Ann. Chem., 544, 163-182 (1940).

6) Kikkawa, H.: Advances in Genetics, 5, 107 (1953).

7) Frey-Wyssling, A.: Submicroscopic Morphology of Protoplasm (1953).

8) Rosenthaler, L.: Der Nachweis organischer Verbindungen, 503-504 (1923).

9) Hopkins, F. G.: Proc. Roy. Soc., 57, No. 5, 6 (1895).

10) Drawert, H.: Planta, 36, 579-600 (1948).

11) Whiting, P. W. and Whiting, A. R.: Biol. Bull., 52, 89-121 (1927). Whiting, P. W., Greb, R. J., and Speicher, B. R.: Biol. Bull., 66, 152-165 (1934).

12) Hannah, A.: J. Exp. Zool., 123, No. 3, 523-555 (1953).

13) Goldschmidt, R.: Die Sexuellen zwischen Stufen (1930).

14) Umeya, Y.: Invertebrate Hormones (1953) (in Jap.). 